\title{
Oxaliplatin: Hohe Aktivität in allen Stadien des Kolonkarzinoms
}

\author{
Zur Sitzung «Colorectal Cancer Malignancies» unter Leitung \\ von Paulo Marcelo Hoff, Centro Paulista de Oncologia, Sao \\ Paulo, und Leonard Saltz, Memorial Sloan Kettering Cancer \\ Center, New York, und zur Postersitzung «Gastrointestinal \\ Cancer» unter Leitung von Charles D. Blanke, Oregon Health \\ and Science University, Portland, und Eric van Cutsem, \\ University Hospital Gasthuisberg, Leuven.
}

Auf der Basis mehrerer Studien gilt heute beim Kolonkarzinom im UICC-Stadium III eine adjuvante Chemotherapie mit 5-Fluorouracil/Folinsäure (5-FU/FA) über 6 Monate als Standard. Im Vergleich zur alleinigen Tumorresektion lässt sich eine Reduktion der Mortalität um rund 30\% erreichen. Immer noch kontrovers diskutiert wird die adjuvante Therapie dagegen im Stadium II. Laut IMPACT(International Multicenter Pooled Analysis of Colon Cancer Trials)-Analyse profitieren adjuvant behandelte Patienten im Vergleich zu ausschließlich operierten Patienten zwar mit einer Steigerung der GesamtÜberlebensrate um absolut $2 \%$ von der Therapie mit 5-FU/FA (81 vs. $83 \%$ ), allerdings war der erzielte Unterschied statistisch nicht signifikant [8]. Auch die vom National Surgical Adjuvant Breast and Bowel Project (NSABP) erstellte gepoolte Analyse vier kleinerer Studien ergab einen nur moderaten Vorteil zugunsten der adjuvanten Therapie im Stadium II [12]. Daher wird die adjuvante Therapie in diesem Stadium bislang bevorzugt bei Patienten mit höherem Rezidivrisiko eingesetzt.

\section{MOSAIC: Was bringt Oxaliplatin in der adjuvanten Therapie?}

Beim inoperablen und metastasierten Kolorektalkarzinom hat die Intensivierung der Chemotherapie durch zusätzliche Gabe neuer Zytostatika wie Oxaliplatin $\left(\right.$ Eloxatin $^{\circledR}$ ) und Irinotecan zu 5-FU/FA-Regimes in den vergangenen Jahren zu einer ausgeprägten Steigerung der Ansprechraten und einer Überle- bensverlängerung bis über 20 Monate hinaus geführt. Die Hoffnung, auch in der adjuvanten Situation mit neuen Kombinationen eine Verbesserung der Therapieergebnisse zu erreichen, konnte jetzt erstmals bestätigt werden: Die auf der 39 . Jahrestagung der American Society of Clinical Oncology (ASCO) vorgestellte internationale MOSAIC(Multicenter International Study of Oxaliplatin/5-FU-LV in the Adjuvant Treatment of Colon Cancer)-Studie ist die erste von mehreren noch laufenden adjuvanten Therapiestudien, in der ein Kombinationsschema - in diesem Fall ein 5-FU/FA-Infusionsregime plus Oxaliplatin - zu einer signifikanten Senkung der Rezidivrate und damit zu einer Verlängerung des krankheitsfreien Überlebens führte [4]. Nach Aussage von Studienleiter Aimery de Gramont, Hôpital Saint Antoine, Paris, sind diese Ergebnisse ein wesentlicher Fortschritt in dem Bemühen, eine größere Zahl von Patienten mit Kolonkarzinom zu heilen. Eine Ablösung des alten adjuvanten Therapiestandards 5-FU/FA durch das effektivere Oxaliplatin-haltige Regime im klinischen Alltag ist nach seinen Worten bereits heute, vor Beendigung der noch laufenden NSABP-Studie mit Oxaliplatin, gerechtfertigt.

In der MOSAIC-Studie wurden 2246 Patienten mit Kolonkarzinomen der UICC-Stadien II (T3, T4, N0, M0) und III (jedes $\mathrm{T}, \mathrm{N} 1, \mathrm{~N} 2, \mathrm{M} 0)$ nach R0-Resektion randomisiert entweder dem Protokoll nach de Gramont (200 mg FA über 2 h i.v., 400 $\mathrm{mg} / \mathrm{m}^{2}$ 5-FU als Bolus, $600 \mathrm{mg} / \mathrm{m}^{2}$ 5-FU i.v. über $22 \mathrm{~h}$, Tage 1 und 2) oder dem gleichen Schema plus Oxaliplatin $\left(85 \mathrm{mg} / \mathrm{m}^{2}\right.$ an Tag 1; FOLFOX4) zugeteilt. Die Charakteristika der Studienteilnehmer sind in Tabelle 1 zusammengefasst. Primärer Endpunkt der MOSAIC-Studie war das krankheitsfreie Überleben; als sekundäre Endpunkte wurden Gesamt-Überleben und Sicherheit definiert. Die Therapie wurde 14-tägig über 12 Zyklen, d.h. über insgesamt 6 Monate, verabreicht. Im FOLFOX4-Arm erhielten 74,7\%, im Kontrollarm 86,5\% der Patienten alle geplanten Therapiezyklen. Im Mittel konnten 81\% der geplanten Oxaliplatin-Dosis appliziert werden.

\section{KARGER}

Fax +497614520714

(C) 2003 S. Karger GmbH, Freiburg

E-mail Information

www.karger.com 
Tab. 1. MOSAIC-Studie: Charakteristika der Teilnehmer im FOLFOX4-Arm und im Kontrollarm (De-Gramont-Schema) [4]

\begin{tabular}{lll}
\hline & $\begin{array}{l}\text { FOLFOX4 } \\
(\mathrm{n}=1123)\end{array}$ & $\begin{array}{l}\text { De-Gramont-Schema } \\
(\mathrm{n}=1123)\end{array}$ \\
\hline Medianes & & \\
Alter, Jahre & 61 & 60 \\
Weibl./männl., \% & $56 / 44$ & $52 / 48$ \\
KPS 80-100\% & 86,2 & 87,6 \\
Stadium II/III, \% & $40 / 60$ & $40 / 60$ \\
Darmobstruktion, \% & 18 & 19 \\
Perforation, \% & 7 & 7 \\
\hline
\end{tabular}

\section{Signifikante Reduktion des Rezidivrisikos um 23\%}

Die Studieninitiatoren gingen bei der Erstellung des Protokolls von der Annahme aus, dass die zusätzliche Gabe von Oxaliplatin zur Standardtherapie mit 5-FU/FA in einer 25\%igen Reduktion der Rezidivrate innerhalb von 3 Jahren resultieren wird - eine Hypothese, die zweifelsfrei verifiziert werden konnte. Während des medianen Follow-up von 37 Monaten wurden im Kontrollarm 288, im FOLFOX4-Arm dagegen nur 234 Rezidive und Todesfälle dokumentiert. Damit war die Rate des krankheitsfreien Überlebens unter dem Oxaliplatinhaltigen Regime mit 77,8\% signifikant höher als bei alleiniger 5-FU/FA-Therapie mit lediglich 72,9\% ( $<<0,01)$. Dieser Unterschied entspricht einer klinisch relevanten Reduktion der Rezidivrate um absolut 5\% und um relativ $23 \%$ durch das FOLFOX4-Regime (Abb. 1). Die höhere Aktivität der Kombination machte sich bereits kurz nach Studienbeginn bemerkbar, wie die von Anfang an auseinanderlaufenden Ereigniskurven der beiden Studienarme verdeutlichen. Patienten der Stadien II und III profitierten mit einer relativen Risikoreduktion von 18 bzw. 24\% vergleichbar stark. Auch die weitergehende Analyse anhand prognostischer Faktoren ergab durchgängig eine - in der Regel deutliche - Überlegenheit der Oxaliplatin-Kombination: «FOLFOX4 verbesserte die Prognose in den verschiedensten Subgruppen, unabhängig von Alter, Geschlecht, T-Stadium, Lymphknotenstatus, Obstruktion oder Perforation, Differenzierungsgrad oder venöser Invasion», betonte de Gramont.

Die derzeitige Beobachtungszeit von gut 3 Jahren ist noch zu kurz, um einen Einfluss von Oxaliplatin auf das GesamtÜberleben festzustellen. De Gramont betonte jedoch, dass es sich bei der vorgestellten Analyse von MOSAIC um die endgültigen Ergebnisse handelt, die sich grundsätzlich auch bei längerem Follow-up nicht mehr ändern werden.

\section{Zusätzliche Oxaliplatin-Gabe wird gut vertragen}

Neben seiner hohen Wirksamkeit überzeugte das FOLFOX4Regime darüber hinaus durch seine Verträglichkeit: Die zusätzliche Gabe von Oxaliplatin zum De-Gramont-Schema

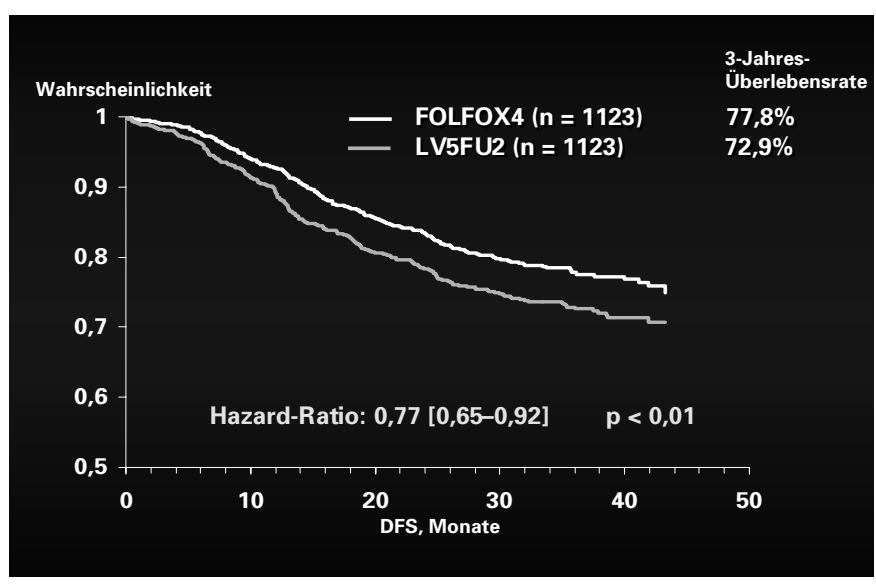

Abb. 1. MOSAIC-Studie: signifikante Steigerung der krankheitsfreien Überlebensrate nach 3 Jahren durch zusätzliche Gabe von Oxaliplatin zur 5-FU/FA-Therapie [4]. Risiko-Reduzierung im FOLFOX-Arm: $23 \%$.

Tab. 2. MOSAIC-Studie: Sicherheitsdaten (Toxizität pro Patient) [4]

\begin{tabular}{lll}
\hline & \multicolumn{2}{l}{ NCI - CTC $\geq$ Gr 3, \% } \\
\cline { 2 - 3 } & $\begin{array}{l}\text { FOLFOX4 } \\
(\mathrm{n}=1108)\end{array}$ & $\begin{array}{l}\text { LV5FU2 } \\
(\mathrm{n}=1111)\end{array}$ \\
\hline Thrombozytopenie & 1,6 & 0,4 \\
Neutropenie & $41,0(\mathrm{Gr} 4: 12,2)$ & 4,7 \\
Febrile Neutropenie & 0,7 & 0,1 \\
Neutr. Sepsis & 1,1 & 0,1 \\
Diarrhö & 10,8 & 6,7 \\
Stomatitis & 2,7 & 2,2 \\
Übelkeit & 5,9 & 1,4 \\
Allergie & 3,0 & 0,2 \\
Alopezie (Gr 2) & 5,0 & 5,0 \\
Gesamtmortalität & 0,5 & 0,5 \\
\hline
\end{tabular}

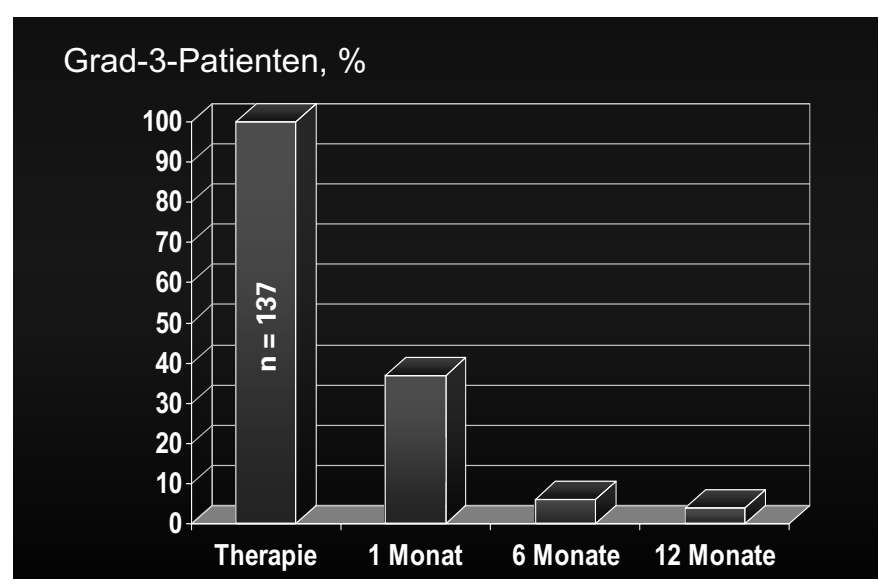

Abb. 2. MOSAIC-Studie: Rasche Rückbildung der Oxaliplatin-bedingten Neuropathie (Grad 3, $\mathrm{n}=137$ ) nach Therapieende [4].

wurde im Allgemeinen gut toleriert. Die Gesamtmortalität war in beiden Studienarmen mit 0,5\% gleich niedrig (Tab. 2). Die gastrointestinale Toxizität bezeichnete de Gramont als 
moderat; eine Alopezie (Grad 2) trat in beiden Armen bei nur $5 \%$ der Patienten auf. Häufiger mit dem FOLFOX4-Regime als im Kontrollarm waren Neutropenien (41 vs. 4,7\%); eine febrile Neutropenie wurde jedoch bei lediglich $0,7 \%$ der Teilnehmer registriert (Kontrollarm: 0,1\%).

Eine periphere sensorische Neuropathie (Grad 3) als hauptsächliche Nebenwirkung von Oxaliplatin entwickelten nur 12,4\% der Patienten im FOLFOX4-Arm. In den meisten Fällen war diese rasch rückläufig: Innerhalb eines Monats nach Therapieende hatte sie sich bereits bei über $60 \%$ der Betroffenen zurückgebildet. Nur 1\% litt noch ein Jahr später unter dieser Nebenwirkung (Abb. 2).

\section{Aktualisierte Ergebnisse zur First-line-Therapie mit Oxaliplatin}

Die auf der ASCO-Tagung 2002 vorgestellte Zwischenanalyse der vom National Cancer Institute (NCI) unterstützten PhaseIII-Intergroup-Studie N9741 beim metastasierten kolorektalen Karzinom hatte die signifikante Überlegenheit des FOLFOX4-Regimes gegenüber dem so genannten Saltz-Regime mit Irinotecan deutlich gemacht [5]. Aufgrund dieser spektakulären Ergebnisse konnte Oxaliplatin nach einem beschleunigten Zulassungsverfahren bald darauf auch für US-Patienten zur Verfügung gestellt werden. Auf der diesjährigen ASCO-Tagung präsentierte Richard M. Goldberg, Mayo Clinic, Rochester, die endgültigen Ergebnisse der Studie N9741 nach mittlerweile 20-monatigem Follow-up [6]. Sie bestätigen eindrucksvoll die anhaltende Überlegenheit der Oxaliplatinhaltigen Kombination: Diese äußert sich in einer Überlebensverlängerung um rund 5 Monate im Vergleich zum Saltz-Regime, dem früheren US-Standard in der First-line-Therapie des fortgeschrittenen Kolorektalkarzinoms, bei gleichzeitig besserer Verträglichkeit.

In der dreiarmigen NCI-Studie wurden Effektivität und Sicherheitsprofil von 3 Kombinationsregimes bei 795 Patienten mit inoperablem, histologisch gesicherten fortgeschrittenen Kolorektalkarzinom geprüft:

Im Kontrollarm erhielten 264 Teilnehmer das Saltz- oder IFLRegime mit $125 \mathrm{mg} / \mathrm{m}^{2}$ Irinotecan plus $500 \mathrm{mg} / \mathrm{m}^{2}$ 5-FU/20 $\mathrm{mg} / \mathrm{m}^{2} \mathrm{FA}$ als Bolus.

267 Patienten wurden mit dem FOLFOX4-Schema behandelt. In einem weiteren experimentellen Arm (IROX) mit $264 \mathrm{~Pa}$ tienten wurde die Kombination Oxaliplatin $\left(85 \mathrm{mg} / \mathrm{m}^{2}\right)$ plus Irinotecan $\left(200 \mathrm{mg} / \mathrm{m}^{2}\right)$ geprüft.

Primärer Studienendpunkt war die Zeit bis zur Progression; sekundäre Endpunkte waren Gesamt-Überleben, Ansprechrate, Toxizität und Lebensqualität.

\section{Überlebensverlängerung auf 20 Monate}

Für nahezu alle Studienendpunkte berichtete Goldberg signifikante Unterschiede zugunsten des FOLFOX4-Regimes im Vergleich zum IFL-Arm: Die Gesamt-Überlebenszeit konnte von 14,8 Monaten bei den mit IFL behandelten Patienten auf 19,5 Monate unter der Oxaliplatin-Kombination verlängert werden ( $\mathrm{p}=0,0001 ;$ Abb. 3). Auch mit der IROX-Kombination behandelte Teilnehmer überlebten mit 17,4 Monaten signifikant länger als Patienten im Kontrollarm $(\mathrm{p}=0,04)$. «Die hohe Effektivität des FOLFOX4-Regimes zeigte sich schon früh im Studienverlauf anhand der bereits kurz nach Therapiebeginn auseinander laufenden Überlebenskurven», betonte Goldberg. Entsprechend war die 1-Jahres-Überlebensrate mit 72\% im FOLFOX4-Arm deutlich höher als unter IFL mit nur 59\% (IROX: 67\%). Die im FOLFOX4-Arm erreichte Verlängerung der Gesamt-Überlebenszeit auf fast 20 Monate ist laut Goldberg die ausgeprägteste Zunahme, die je in einer US-Studie beobachtet wurde.

Auch hinsichtlich der Ansprechraten schnitten FOLFOX4und IROX-Regime signifikant besser ab als das Saltz-Protokoll (Tab. 3). Darüber hinaus profitierten die Patienten von der höheren Aktivität des FOLFOX4-Regimes in Form einer signifikanten Verlängerung der progressionsfreien Zeit um gut 2 Monate im Vergleich zum IFL-Arm (8,7 vs. 6,9 Monate; $\mathrm{p}=0,0014)$.

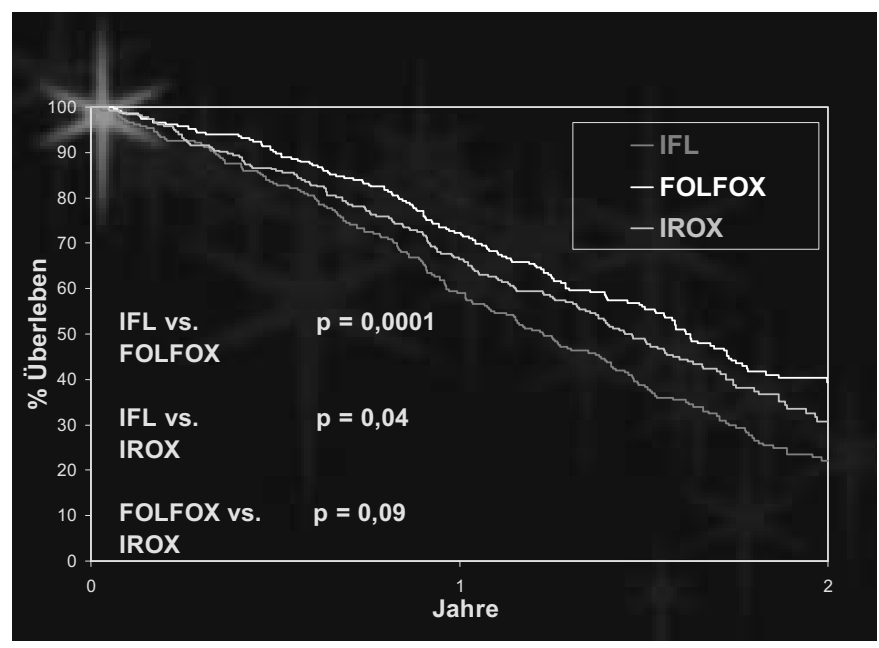

Abb. 3. NCI-Studie N9741: Kaplan-Meier-Kurven für das GesamtÜberleben in den 3 Studienarmen [6].

Tab. 3. NCI-Studie N9741: Ergebnisse der aktualisierten Analyse [6]

\begin{tabular}{lccc}
\hline & IFL & FOLFOX & IROX \\
\hline OS, Monate & 14,8 & 19,5 & 17,4 \\
TTP, Monate & 6,9 & 8,7 & 6,5 \\
RR, \% & 31 & 45 & 34 \\
\hline
\end{tabular}

OS = Gesamt-Überleben; RR = Ansprechrate;

TTP = Zeit bis zur Progression. 


\section{FOLFOX4: Neuer Standard in der First-line-Therapie}

Mit Ausnahme der peripheren Neuropathie war die Toxizität des FOLFOX4-Regimes generell signifikant geringer als die des Saltz-Regimes: Febrile Neutropenien, Diarrhöen, Übelkeit und Erbrechen sowie Alopezie traten unter der Oxaliplatin-Therapie seltener auf bzw. verliefen weniger schwer. Dies resultierte jedoch nicht in signifikanten Unterschieden in der allgemeinen Lebensqualität zwischen den Studienarmen. Ursächlich könnten laut Goldberg die langen Intervalle von 12 Wochen zwischen den einzelnen Erhebungszeitpunkten sein, so dass die Auswirkungen akut auftretender Nebenwirkungen nicht erfasst werden. Insgesamt bewertete er das FOLFOX4Regime im Vergleich zum IFL-Regime als bisherigem USTherapiestandard eindeutig als vorteilhaft: «Aufgrund der signifikanten Überlegenheit bei allen relevanten Studienendpunkten und des günstigeren Toxizitätsprofils ist FOLFOX4 von nun an als neuer Standard in der First-line-Therapie des metastasierten kolorektalen Karzinoms zu betrachten», resümierte Goldberg.

«Trotz mehrerer Protokolländerungen im Studienverlauf ist die NCI-Studie N9741 eine qualitativ gute Untersuchung von klinisch hoher Relevanz, die das Vorgehen im praktischen Alltag ändern wird», erklärte auch Eric van Cutsem, University Hospital Gasthuisberg, Leuven, in seiner Diskussion der Ergebnisse. (Die Protokolländerungen wurden nötig, da 3 der ursprünglich 6 Arme der NCI-Studie wegen geringerer Wirksamkeit und/oder höherer Toxizität vorzeitig abgebrochen werden mussten.) Darüber hinaus hat die Studie nachdrücklich klar gemacht, dass die in Europa bevorzugten 5-FU-Infusionsregimes aufgrund ihrer geringeren Toxizität als Basis für Kombinationen wesentlich geeigneter sind als die in den USA hauptsächlich eingesetzten Bolusregimes. Ein Vergleich aller bislang mit Kombinationsregimes durchgeführten Studien weist außerdem darauf hin, so van Cutsem, dass betroffenen Patienten möglichst alle 3 beim Kolorektalkarzinom aktiven Zytostatika - 5-FU, Oxaliplatin und Irinotecan - zur Verfügung gestellt werden sollten, um das Überleben optimal zu beeinflussen. Als wichtige Herausforderung für die Zukunft betrachtet er die gezielte Evaluierung von Kombinationstherapien, in denen 5-FU/FA durch orale 5-FU-Prodrugs wie Capecitabin ersetzt wird.

\section{Cap0x: Hohe Ansprechraten, gute Verträglichkeit}

Dies geschieht derzeit in einer deutschen Phase-II-Studie an 161 nicht vorbehandelten Patienten mit metastasiertem oder inoperablem Kolorektalkarzinom [7]. Sie wurden randomisiert einer Therapie mit Capecitabin $\left(1000 \mathrm{mg} / \mathrm{m}^{2} 2 \times / \mathrm{Tag}\right.$, Tage 1-14) plus Irinotecan $\left(100 \mathrm{mg} / \mathrm{m}^{2}\right.$ i.v. Tage 1 und 8$)$ oder der Kombination mit Capecitabin plus Oxaliplatin $\left(70 \mathrm{mg} / \mathrm{m}^{2}\right.$ i.v. Tage 1 und 8) zugeteilt. Die beiden als CapIri bzw. CapOx bezeichneten Protokolle erwiesen sich als hoch effektiv: Im Ca-
Tab. 4. Therapieergebnisse mit den Kombinationen Capecitabin/Irinotecan (CapIri) und Capecitabin/Oxaliplatin (Cap/Ox) in einer deutschen Multizenterstudie [7]

\begin{tabular}{lccc}
\hline & CapIri & CapOx & p-Wert \\
\hline CR, \% & 2,9 & 6,6 & \\
PR, \% & 39,7 & 44,7 & \\
SD, \% & 39,7 & 39,5 & \\
PD, \% & 17,6 & 9,2 & \\
CR/PR, \% & 42,6 & 51,3 & 0,34 \\
\hline
\end{tabular}

$\mathrm{CR}=$ Komplette Remission; $\mathrm{PR}=$ partielle Remission; $\mathrm{SD}=$ Stabilisierung; $\mathrm{PD}=$ Progression

pIri-Arm lag die Gesamtresponserate bei 42,6\%; 2,9\% der Patienten sprachen komplett an. Mit dem CapOx-Regime wurde eine Ansprechrate von insgesamt 51,3\%, davon 6,6\% Komplettremissionen, dokumentiert (Tab. 4). Darüber hinaus gelang in beiden Armen bei knapp 40\% der Patienten eine Tumorstabilisierung. Das progressionsfreie Intervall war mit 7,9 Monaten für beide Protokolle identisch.

Nach Progression erfolgte ein Cross-over zum jeweils anderen Regime: Nach vorläufigen Daten an insgesamt 52 Patienten führte die Second-line-Therapie mit CapIri bei 18,5\% der Patienten zu Remissionen, bei weiteren 55,6\% zur Stabilisierung. Auf das CapOx-Regime als Zweitlinientherapie sprachen $12 \%$ der Patienten an (8\% partielle, $4 \%$ komplette Remissionen). Eine Stabilisierung wurde bei $44 \%$ erreicht.

Grothey et al. [7] beschreiben die Inzidenz der Grad 3/4-Toxizität beider Regimes als vergleichbar. Die Hämatotoxizität war relativ schwach ausgeprägt. Allerdings verstarben 4 der ersten 40 Patienten des CapIri-Arms innerhalb von 60 Tagen nach Therapiebeginn, so dass die Irinotecan-Dosis in der Folge ohne Einbuße an Effektivität - auf $80 \mathrm{mg} / \mathrm{m}^{2}$ gesenkt wurde. Insgesamt lag die 60-Tage-Gesamtmortalität im CapIri-Arm bei 6,3\%, im CapOx-Arm dagegen bei nur 1,2\%. Das sehr effektive und dabei gut verträgliche CapOx-Regime wird auf der Basis dieser Daten jetzt in einer Phase-III-Studie der Arbeitsgemeinschaft internistischer Onkologen (AIO), für die $420 \mathrm{~Pa}-$ tienten rekrutiert werden sollen, mit der FUFOX-Kombination aus Oxaliplatin plus AIO-Infusionsregime verglichen.

\section{OPTIMOX: Optimierung des Oxaliplatin-Regimes}

Ähnlich wie das deutsche FUFOX-Schema ist auch das französische Kombinationsregime FOLFOX4 als hoch effektiv und insgesamt gut verträglich bekannt. Dosislimitierend ist zum einen die mit Oxaliplatin mögliche kumulative sensorische Neuropathie, die einen Behandlungsabbruch bei ansprechenden Patienten erforderlich machen kann. Darüber hinaus kann der 14-tägig verabreichte 5-FU-Bolus eine Neutropenie induzieren. Um die Verträglichkeit der Oxaliplatin-haltigen Kombinationstherapie weiter zu verbessern, wird in der OP- 


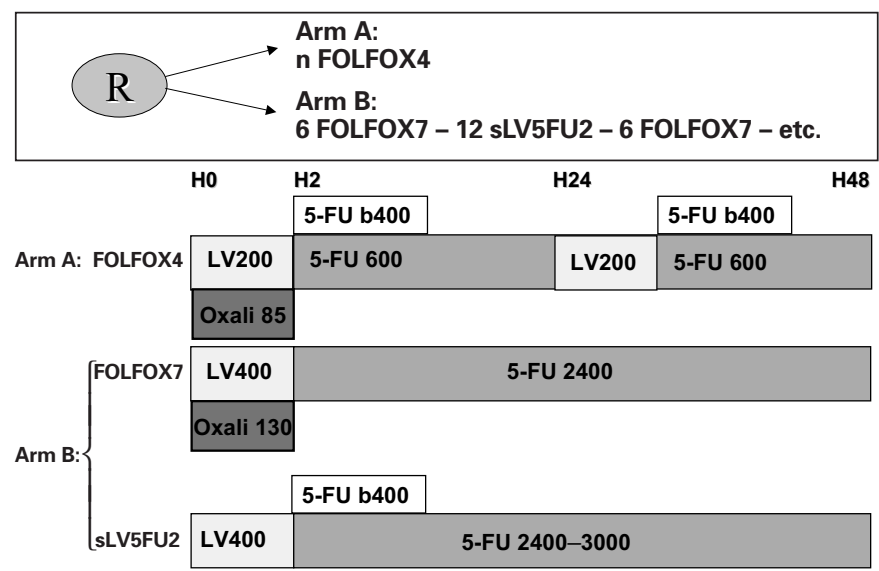

Abb. 4. Design der OPTIMOX-Studie [2].

TIMOX-Studie das FOLFOX7-Schema geprüft. Hier wird eine höhere Oxaliplatindosis von $130 \mathrm{mg} / \mathrm{m}^{2}$ mit einem 5FU/FA-Regime ohne Bolusgabe kombiniert. Nach 6 Gaben FOLFOX7 wird dann mit einem vereinfachten 5FU/FA-Regime (s5LV5FU2) ohne Oxaliplatin weiterbehandelt. Nach 12 Zyklen, oder bei Progression schon früher, wird wieder auf FOLFOX7 umgestellt.

Für die europäische Studie wurden 623 Patienten mit histologisch gesichertem, inoperablem oder metastasiertem Kolorektalkarzinom randomisiert entweder dem FOLFOX4-Regime (Arm A) oder dem Arm B zugeteilt (Abb. 4).

Im Follow-up von median 13 Monaten zeigten beide Therapieregimes eine vergleichbare Effektivität: Mit dem FOLFOX4-Regime wurde eine Gesamtresponserate von 59,8\% erzielt; 7,4\% der Patienten sprachen komplett, 52,5\% partiell an. Im Arm B lag die Gesamtresponserate bei $63 \%$. In 10,8\% der Fälle handelte es sich um komplette, in 52,3\% um partielle Remissionen. Auch das progressionsfreie Überleben war mit rund 9 Monaten in beiden Armen vergleichbar. Um diese Ergebnisse zu erreichen, wurden im Arm A median 11, im Arm B median 7 Oxaliplatin-haltige Zyklen verabreicht.

Neutropenien vom Grad 3/4 und eine Neuropathie vom Grad 3 wurden mit dem FOLFOX7-Regime - wie erhofft - signifikant seltener registriert als mit FOLFOX4 ( $p=0,009$ bzw. $p=$ 0,0017 ). Darüber hinaus schätzen die Autoren das FOLFOX7Regime auch als patientenfreundlicher ein. Allerdings traten im Arm B häufiger verzögerte Thrombozytopenien (Grad 3/4) auf. In der bereits laufenden OPTIMOX-2-Studie wird derzeit der Arm B der vorgestellten OPTIMOX-Studie (mit FOLFOX7 - s5-FU/FA - FOLFOX7) als Kontrollarm im Vergleich zur alleinigen FOLFOX7-Applikation geprüft.

\section{Ausgeprägte Palliation in der Second-line-Therapie}

Vor der Zulassung von Oxaliplatin in den USA erhielten rund $70 \%$ aller Patienten mit fortgeschrittenem Kolorektalkarzinom eine First-line-Therapie mit dem IFL-Regime. Kommen diese Patienten trotz Therapie in den Progress, hat sich das FOLFOX4-Regime mit Oxaliplatin als wichtige Option zur Verzögerung der Progression und zur Linderung tumorbedingter Symptome wie Gewichtsverlust, Schmerzen, Fatigue und Schwäche erwiesen, resümierte Mace L. Rothenberg, Vanderbilt-Ingram Cancer Center, Nashville, die Endresultate der Studie EFC 4584 [13]. Für die dreiarmige Phase-III-Studie waren 821 unter dem Saltz-Regime progrediente Patienten randomisiert einer Therapie mit 5-FU/FA als Bolus oder Infusion, einer Monotherapie mit Oxaliplatin $\left(85 \mathrm{mg} / \mathrm{m}^{2}\right)$ oder dem FOLFOX4-Regime zugeteilt worden. Etwa zwei Drittel der Teilnehmer waren bei Therapiebeginn symptomatisch. Primärer Studienendpunkt war das Gesamt-Überleben; als sekundäre Endpunkte wurden Ansprechrate, Zeit bis zur Progression, Zeit bis zur symptomatischen Verschlechterung und Sicherheit definiert.

Laut der von Rothenberg präsentierten Analyse der Studiendaten schneidet das FOLFOX4-Regime bei nahezu allen Wirkparametern signifikant besser ab als die beiden anderen Studienarme: 9,6\% der Patienten im FOLFOX4-Arm sprachen auf die Therapie an. In den Vergleichsarmen mit 5FU/FA bzw. Oxaliplatin betrug die Responserate nur 0,7 bzw. $1,1 \%$. Die Zeit bis zur Progression wurde durch die Oxaliplatin-Kombination auf 5,6 Monate verlängert und war damit rund 3 Monate länger als im 5-FU/FA-Arm mit nur 2,6 Monaten und unter der Oxaliplatin-Monotherapie mit 1,9 Monaten. Als eindrucksvoll bezeichnete Rothenberg die ausgeprägte Symptomlinderung durch das FOLFOX4-Regime bei $28 \%$ der Patienten. Im 5-FU/FA-Arm besserten sich die Symptome bei $15 \%$, im Oxaliplatin-Arm bei 10\% (Tab. 5). Die Gesamt-Überlebenszeit ab Second-line-Therapie mit dem FOLFOX4-Regime betrug 9,8 Monate und war damit nicht signifikant länger als unter 5-FU/FA (8,7 Monate) und Oxaliplatin (8,1 Monate). Laut Rothenberg könnte die fehlende Signifikanz unter anderem darauf beruhen, dass über die Hälfte der Patienten im 5-FU/FA-Arm nach Progress noch eine Salvage-Therapie mit Oxaliplatin erhielten.

Diarrhöen, Übelkeit/Erbrechen, Neutropenien und Neuropathien wurden unter dem FOLFOX4-Regime häufiger registriert als in den Vergleichsarmen. Diese Nebenwirkungen bezeichnete Rothenberg jedoch als vorhersehbar und einfach zu kontrollieren.

Auch wenn die Überlebenszeit als primärer Endpunkt dieser Studie durch das FOLFOX4-Schema nicht signifikant verlängert werden konnte, darf dieses Regime progredienten Patienten nicht vorenthalten werden, kommentierte van Cutsem. Denn gerade in der palliativen Situation klinisch relevante Endpunkte wie progressionsfreies Intervall und Linderung tumorassoziierter Symptome werden durch die OxaliplatinKombination deutlich verbessert. 
Tab. 5. Studie zur Second-line-Therapie: Stärkere Verbesserung tumorbedingter Symptome mit dem FOLFOX4-Regime vs. 5-FU/FA vs. Oxaliplatin-Monotherapie [13]. n.s. = Nicht signifikant

\begin{tabular}{llll}
\hline Parameter & \multicolumn{2}{l}{ Verbesserung, $\%$} & $\begin{array}{l}\text { p-Wert } \\
\left(\chi^{2} \text { Test }\right)\end{array}$ \\
\cline { 2 - 3 } & $\begin{array}{llll}\text { LV5FU } \\
(\mathrm{n}=171)\end{array}$ & $\begin{array}{l}\text { Oxaliplatin } \\
(\mathrm{n}=163)\end{array}$ & $\begin{array}{l}\text { FOLFOX } \\
(\mathrm{n}=167)\end{array}$ \\
\hline
\end{tabular}

Karnofski-Performance-

\begin{tabular}{|c|c|c|c|c|}
\hline Status & 3 & 4 & 9 & \\
\hline Schmerz & 24 & 14 & 40 & \\
\hline Analgetika-Verbrauch & 9 & 8 & 32 & \\
\hline Gewichtsverlust & 8 & 2 & 10 & \\
\hline Kombination & $\{15$ & - & 28 & $<0,002$ \\
\hline & 15 & 10 & - & n.s. \\
\hline
\end{tabular}

\section{OXALIPLATIN: Neue Therapieoption auch bei Pankreas- und Magenkarzinom}

Zur Sitzung «Gastrointestinal (Non-Colorectal) Cancer unter Leitung von Jordan Berlin, Vanderbilt-Ingram Comprehensive Cancer Center, Nashville, und Charles R. Thomas, University of Texas Health Science Center, San Antoni, zur Sitzung «Gastrointestinal Cancer» unter Leitung von James L. Abbruzzese, und Steven A. Curley, University of Texas M.D. Anderson Cancer Center, Houston, und zur Postersitzung «Gastrointestinal Cancer».

Bereits 2001 hatte eine während der ASCO-Tagung vorgestellte und mittlerweile publizierte Phase-II-Studie auf das vielversprechende Potenzial von Gemcitabin $\left(1000 \mathrm{mg} / \mathrm{m}^{2}\right.$ als 100-min-Infusion, Tag 1) plus Oxaliplatin $\left(100 \mathrm{mg} / \mathrm{m}^{2}\right.$ i.v., Tag 2) alle 2 Wochen beim fortgeschrittenen Pankreaskarzinom aufmerksam gemacht [10]. Die beeindruckende Aktivität der als GEMOX bezeichneten Kombination bei diesem nur schwer therapierbaren Tumor zeigte sich anhand der hohen Ansprechrate von 31\% und einer klinischen Benefit-Rate von $39,7 \%$. Erreicht wurden ein medianes progressionsfreies Intervall von 5,3 und eine Gesamt-Überlebenszeit von 9,2 Monaten. Die 1-Jahres-Überlebensraten von 47\% bei Patienten mit lokal fortgeschrittenem und von $26 \%$ beim metastasierten Pankreaskarzinom in dieser Studie lagen deutlich über denen der Gemcitabin-Monotherapie als derzeitigen Therapiestandard (Abb. 5). Darüber hinaus zeichnet sich die GEMOX-Kombination durch ein günstiges Verträglichkeitsprofil aus.

\section{GEMOX: Viel versprechende Kombination bei Pankreaskrebs}

Diese ermutigenden Daten rechtfertigten die Initiierung einer Phase-III-Studie, in der die neue Kombination mit Gemcitabin als Monotherapie verglichen wurde. Die ersten Resultate

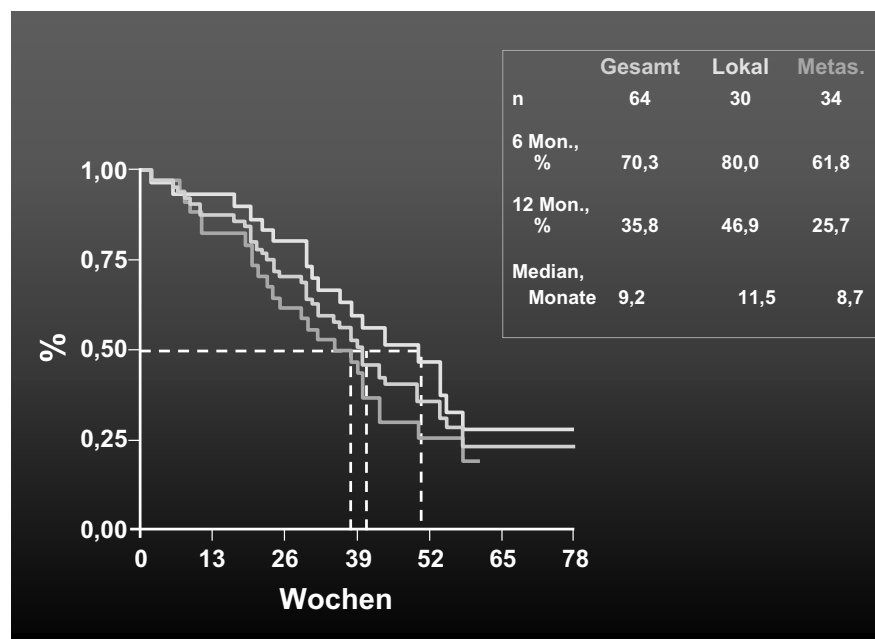

Abb. 5. Phase-II-Studie beim Pankreaskarzinom: Überleben im Gesamtkollektiv und in den Subgruppen von Patienten mit lokal fortgeschrittenem und metastasierten Pankreaskarzinom [10].

Lokal $=$ lokal fortgeschritten; Metas. $=$ metastasiert; Mon. $=$ Monate .

dieser Untersuchung, die Christophe Louvet, Hôpital Saint Antoine, Paris, auf der ASCO-Tagung 2003 vorstellte, bestätigen die Daten der Phase-II-Studie und sprechen für eine stärkere Tumorschrumpfung, eine Verlängerung des progressionsfreien Intervalls und einen vermehrten klinischen Benefit durch zusätzliche Gabe von Oxaliplatin zur Gemcitabin-Therapie [11]. Für die von der französischen GERCOR- und der italienischen GISCAD-Gruppe durchgeführte Studie wurden zwischen März 2001 und Februar 2003326 Patienten mit histologisch gesichertem Pankreasadenokarzinom rekrutiert. 156 Patienten wurden randomisiert dem Kontrollarm mit Gemcitabin ( $1 \mathrm{~g} / \mathrm{m}^{2}$ wöchentlich als 30-minütige Infusion), $157 \mathrm{~Pa}$ tienten dem experimentellen Arm mit der GEMOX-Kombination zugeteilt. Etwa 30\% der Patienten in beiden Armen wiesen einen lokal fortgeschrittenen, $70 \%$ einen metastasierten Tumor auf. Primäres Ziel der Studie war eine Zunahme der Überlebenszeit von 6 auf 8 Monate. Sekundäre Zielparameter waren progressionsfreies Überleben, Ansprechrate, klinischer Benefit, Verträglichkeit und Lebensqualität.

Zum Zeitpunkt der Analyse im Mai 2003 lebten noch 44,5\% der rekrutierten Teilnehmer, so dass es für eine endgültige Analyse von Überlebensdaten noch zu früh ist, erläuterte Louvet. $81 \%$ der Patienten im Kontrollarm und $73 \%$ der Patienten im GEMOX-Arm sind mittlerweile progredient; die Ergebnisse zum progressionsfreien Überleben sind daher als endgültig zu betrachten. Patienten im Gemcitabin-Arm erhielten median 7 Zyklen, Patienten im GEMOX-Arm 6 Zyklen. Im Mittel konnten rund $90 \%$ der geplanten Dosis verabreicht werden.

Auch in dieser Phase-III-Studie überzeugte die GEMOXKombination durch eine hohe Aktivität: 25,8\% der Patienten sprachen partiell auf die Oxaliplatin-haltige Therapie an, signifikant mehr als im Vergleichsarm mit einer Rate von 16,1\% $(\mathrm{p}=0,03)$, die Louvet als erstaunlich hoch für die Gemcitabin- 
Tab. 6. Phase-III-Studie mit GEMOX versus Gemcitabin beim fortgeschrittenen Pankreaskarzinom [11]

\begin{tabular}{llll}
\hline & Gemcitabin & GEMOX & p-Wert \\
\hline PR, \% & 16,1 & 25,8 & 0,03 \\
PFS, Wochen & & & \\
- alle Patienten & 16 & 25 & \\
- lokal fortgeschritten & 23 & 38 & \\
- metastasiert & 14 & 20 & 0,05 \\
Klinischer Benefit, \% & 28,3 & 39,2 & \\
\hline
\end{tabular}

$\mathrm{PR}=$ Partielle Remission; PFS = progressionsfreies Überleben.

Monotherapie bezeichnete (Tab. 6). Der Vorteil der Kombination hinsichtlich der Responsequoten galt sowohl für Patienten mit lokal fortgeschrittenem als auch mit metastasiertem Pankreaskarzinom. Die Ansprechdauer war mit gut 40 Wochen in beiden Studienarmen vergleichbar.

\section{GEMOX aktiver als Gemcitabin-Standardtherapie}

Ein deutlicher Vorteil zugunsten der Kombination zeigte sich hinsichtlich des progressionsfreien Überlebens: Mit GEMOX behandelte Patienten überlebten median 25,8 Wochen ohne Progress, während dieses Intervall im Kontrollarm nur 16 Wochen betrug. Sowohl Patienten mit lokal fortgeschrittenem als auch mit metastasiertem Tumor profitierten von der Oxaliplatin-Kombination mit einer Verlängerung des progressionsfreien Überlebens. Auch die klinische Benefit-Rate, mit der Verbesserung von Allgemeinzustand und Lebensqualität sowie Verminderung von Schmerzen erfasst werden, war im GEMOX-Arm mit 39,2\% deutlich höher als im GemcitabinArm (28,3\%; $\mathrm{p}=0,05)$. Dieser Unterschied zugunsten von GEMOX zeigte sich ebenfalls in beiden Patientensubgruppen. Wie Louvet betonte, entsprachen Response- und klinische Benefit-Raten dieser Phase-III-Studie etwa der der vorausgegangenen Phase-II-Studie, obwohl das Studienkollektiv aufgrund des höheren Anteils von Patienten mit metastasiertem Tumor prognostisch deutlich ungünstiger war. Er hofft, dass die höhere Aktivität der GEMOX-Kombination beim Pankreaskarzinom letztlich auch in einer Überlebensverlängerung resultieren wird.

Beide Therapieregimes wurden ähnlich gut vertragen. Häufiger mit der Gemcitabin-Therapie als unter der GEMOXKombination wurden febrile Neutropenien (5,1 vs. 4,5\%) und ein Hämoglobin-Abfall (9,6 vs. 6,4\%) beobachtet. Umgekehrt waren die Raten von Thrombozytopenie, Erbrechen und Neuropathie (Grad 3) bei kombinierter Therapie höher als im Kontrollarm. «Vergleicht man die maximale Toxizität vom Grad 3 und 4 in beiden Armen, so sind die Raten mit 40\% unter Gemcitabin-Monotherapie und 48\% im GEMOX-Arm nicht signifikant unterschiedlich», betonte Louvet.
Margaret A. Tempero, San Francisco Cancer Center, University of California, bezeichnete die französisch-italienische Studie als wichtig und qualifizierte die mit der GEMOX-Kombination erreichte Verlängerung des progressionsfreien Überlebens bis auf 25 Wochen als «aufregend». Unter den verschiedenen, derzeit beim Pankreaskarzinom geprüften Gemcitabin-Kombinationen nehme GEMOX daher die Spitzenstellung ein, so Tempero. Zu prüfen sei jetzt, ob der Vorteil von GEMOX auch im Vergleich zu einer Gemcitabin-Infusion mit fixierter Dosierungsgeschwindigkeit, die in verschiedenen Studien eine hohe Effektivität gezeigt hatte $\left(10 \mathrm{mg} / \mathrm{m}^{2} / \mathrm{min}\right)$, bestehen bleibe. Dieser Frage geht man zur Zeit in einer dreiarmigen Phase-III-Studie (E6201) nach.

\section{Magenkrebs: Auf der Suche nach einem neuen Therapiestandard}

In der palliativen Chemotherapie beim fortgeschrittenen Magenkarzinom gilt bislang noch das ECF-Regime mit $50 \mathrm{mg} / \mathrm{m}^{2}$ Epirubicin, $60 \mathrm{mg} / \mathrm{m}^{2}$ Cisplatin und $200 \mathrm{mg} / \mathrm{m}^{2} / \mathrm{Tag} 5-\mathrm{FU}$ als Referenztherapie. Zwei großen randomisierten Studien zufolge können damit Responseraten zwischen 40 und $45 \%$ und eine mediane Überlebenszeit von rund 9 Monaten erreicht werden.

Allerdings ist das ECF-Regime wegen der dauerhaften Versorgung mit einer Pumpe wenig patientenfreundlich, Alternativen sind daher wünschenswert. In einer Multizenterstudie der Phase III, für die insgesamt 600 therapienaive Patienten mit fortgeschrittenem Magen/Ösophagus-Karzinom rekrutiert werden sollen, wird zur Zeit eine Verbesserung des ECFSchemas durch Ersatz von 5-FU durch Capecitabin und von Cisplatin durch Oxaliplatin erprobt. Die vierarmige Studie besitzt ein faktorielles $2 \times 2$-Design: In den vier Studienarmen wird der ECF-Standard mit den Kombinationen Epirubicin/Oxaliplatin/5-FU (EOF), Epirubicin/Cisplatin/Capecitabin (ECX) und Epirubicin/Oxaliplatin/Capecitabin (EOX) verglichen.

Bereits die erste, auf der ASCO-Tagung 2002 vorgestellte Interimsanalyse der Studie an 80 Patienten hatte für alle vier Regimes eine vielversprechende Aktivität bei nur mäßig ausgeprägter hämatologischer Toxizität aufgezeigt [15]. Für die nächsten 80 Patienten war daher eine Dosiseskalation von 1000 auf $1250 \mathrm{mg} / \mathrm{m}^{2}$ Capecitabin vorgesehen. Gemäß der auf der diesjährigen ASCO-Tagung präsentierten Analyse an 176 evaluierbaren Patienten stieg die Rate der nicht-hämatolgischen Toxizität ( Grad 3/4) von 5,1\% unter der niedrigeren auf 10,7\% mit der höheren Capecitabin-Dosierung (Tab. 7), war damit jedoch immer noch niedriger als unter 5-FU mit 13,3\% [14]. Die Rate partieller und kompletter Remissionen konnte mit dem EOX-Regime, d.h. bei Einsatz der beiden modernen Zytostatika Oxaliplatin und Capecitabin, bis auf 52\% gesteigert werden. In den anderen drei Studienarmen wurden Responseraten zwischen 31 und $35 \%$ ermittelt. 
Tab. 7. Phase-III-Studie beim fortgeschrittenen Magen-ÖsophagusKarzinom: Sicherheitsdaten [14]

\begin{tabular}{lcccccc}
\hline & ECX & EOX & ECX & EOX & ECF & ECF \\
\hline X-Dosis, mg/m² & 1000 & 1000 & 1250 & 1250 & - & - \\
5-FU-assoz. Tox. & 6,9 & & 10,7 & & & \\
Patienten, n & 27 & 31 & 13 & 15 & 46 & 44 \\
Toxizität Grad 3/4, \% & & & & & & \\
Stomatitis & 0 & 0 & 0 & 0 & 8,7 & 0 \\
Diarrhö & 0 & 6,4 & 0 & 6,7 & 8,7 & 2,3 \\
PPE & 3,7 & 3,2 & 15,4 & 0 & 2,2 & 4,6 \\
Neutropenie & 38,5 & 43,8 & 33,3 & 30,8 & 21,7 & 34,1 \\
Febrile Neutrop. & 10 & 0 & 0 & 0 & 7,4 & 9 \\
\hline
\end{tabular}

$\mathrm{E}=$ Epirubicin $\mathrm{O}=$ Oxaliplatin; $\mathrm{X}=$ Capecitabin; $\mathrm{F}=$ 5-Fluorouracil; $\mathrm{PPE}=$ Hand-Fuß-Syndrom.

\section{Oxaliplatin-Kombination effektiv und verträglich}

Al-Batran et al. [1] prüften in einer multizentrischen Phase-IIStudie die Aktivität einer FLO-Kombination aus Oxaliplatin $\left(85 \mathrm{mg} / \mathrm{m}^{2}\right)$ und einem 5-FU/FA-Infusionsregime beim fortgeschrittenen Magenkarzinom. Zwischen November 1999 und September 2002 wurden an 6 deutschen Zentren insgesamt 41 Patienten mit histologisch gesichertem, inoperablem oder metastasiertem Magenkarzinom rekrutiert. 16 der 37 auf Response evaluierbaren Patienten (43,2\%) sprachen auf die Therapie mit der Oxaliplatin-Kombination an, einer davon mit einer kompletten Remission. Bei weiteren 12 Patienten (32\%) gelang eine Tumorstabilisierung. Die Zeit bis zur Progression beträgt 5,6 Monate; das Gesamt-Überleben liegt derzeit bei 9,6 Monaten. Die Aktivität dieser Oxaliplatin-Kombination ist damit beachtenswert und durchaus vergleichbar mit der anderer, in Studien der Phase II und III evaluierten Regimes wie ECF oder FOLFOX6, betonen die Autoren. Die Toxizität der deutschen Oxaliplatin-Kombination ist jedoch wegen der niedrigeren Dosierungen von Oxaliplatin und 5-FU deutlich geringer als die von FOLFOX6. Dies gilt insbesondere für Neutropenie (Grad 3/4) und periphere Neuropathie (Grad 3/4), die unter dem FOLFOX6-Regime deutlich häufiger als mit der FLO-Kombination auftreten (38 vs. 4,9\% bzw. 21 vs. 0\%). Nach Aussage von Al-Batran und Mitarbeitern lassen sich die auf der ASCO-Tagung präsentierten Ergebnisse durch eine multimodale Therapie mit präoperativer Gabe der Oxaliplatin-Kombination und anschließender Resektion und durch den Einsatz im Rahmen sequenzieller Regimes vermutlich noch weiter verbessern.

\section{Refraktäres Hodenkarzinom: Oxaliplatin als neue Hoffnung}

\author{
Zur Postersitzung "Genitourinary (Nonprostate) Cancer» \\ unter Leitung von Pieter H.M. de Mulder, University Medical \\ Center Nijmegen, und Eric A. Klein, Cleveland Clinic \\ Foundation.
}

Patienten mit metastasiertem Hodentumor haben mit einer Cisplatin-haltigen Chemotherapie und anschließender Resektion residueller Metastasen eine hohe Heilungschance von $70-80 \%$. Schlechter ist die Prognose jedoch bei Patienten, die nach einer solchen Kombinationschemotherapie rezidivieren oder bei denen selbst die Hochdosis-Chemotherapie mit peripherem Stammzell-Support versagt. Bei diesen Patienten mit Cisplatin-refraktärem Keimzelltumor haben bislang nur Paclitaxel und Gemcitabin eine gewisse Aktivität mit einer Responserate um 20\% gezeigt. Eine auf der ASCO-Tagung 2001 vorgestellte Phase-II-Studie der German Testicular Cancer Study Group (GTCSG) hatte dann jedoch zeigen können, dass mit Oxaliplatin ein Durchbrechen der Cisplatin-Resistenz beim refraktären Hodenkarzinom und Responseraten um $20 \%$ möglich sind [9].

\section{Gemcitabin/Oxaliplatin: Effektiv bei intensiv vortherapierten Patienten}

Aufgrund dieser hoffnungsvollen Daten initiierte die GTCSG mittlerweile eine weitere Phase-II-Studie, in der die Kombinationstherapie mit Gemcitabin $\left(1000 \mathrm{mg} / \mathrm{m}^{2}\right.$ über 30 Minuten, Tage 1,8$)$ gefolgt von Oxaliplatin $\left(130 \mathrm{mg} / \mathrm{m}^{2}\right.$ an Tage 1,21$)$ evaluiert wird [3]. Bislang wurden 29 Patienten mit Cisplatinrefraktärem Keimzelltumor rekrutiert. Alle Studienteilnehmer waren intensiv vorbehandelt. Sie hatten bereits median 6 platinhaltige Therapiezyklen und $86 \%$ hatten eine Hochdosis-Chemotherapie mit autologer Stammzelltransplantation durchlaufen. Zwei Drittel der Patienten wurden als Cisplatinrefraktär eingestuft.

Elf der 29 Patienten (38\%) sprachen mit einer radiologisch bestätigten partiellen Remission und einem TumormarkerAbfall um mehr als $80 \%$ auf die Kombinationstherapie an. 8 dieser 11 Remissionen wurden bei Cisplatin-refraktären $\mathrm{Pa}$ tienten erreicht. Das mediane progressionsfreie Intervall bei ansprechenden Patienten betrug 3 Monate; 5 Patienten blieben über mehr als 5 Monate in Remission. Die Autoren bezeichnen die Toxizität der ambulant verabreichten Gemcitabin/Oxaliplatin-Kombination als akzeptabel: Neutropenien und Thrombozytopenien vom Grad 3 und 4 waren meist nur von kurzer Dauer und wurden bei 55 bzw. 45\% der Teilnehmer registriert. Eine febrile Neutropenie entwickelten lediglich 3 Patienten. Auch die Neuropathie-Inzidenz war mit 10\% niedrig. Aufgrund der beachtlichen Aktivität der Gemcitabin/Oxaliplatin-Kombination werden weiterhin Patienten in die Phase-II-Studie aufgenommen. 


\section{Literatur}

1 Al-Batran SE, Atmaca A, Hegewisch-Becker S, et al.: Oxaliplatin, 5-fluorouracil and leucovorin every two weeks in patients with advanced gastric cancer; preliminary results of a multicenter, phase II study. Proc ASCO 2003;22:abstr 1128 (poster presentation).

2 André T, Figer A, Cervantes A, et al.: FOLFOX7 compared to FOLFOX4. Preliminary results of the randomized optimox study. Proc ASCO 2003;22: abstr 1016 ( poster presentation).

3 Bokemeyer C, Beyer J, Metzner B, et al.: Combination chemotherapy with gemcitabine/oxaliplatin in patients (pts) with relapsed or cisplatin-refractory germ-cell cancer (GCT). Proc ASCO 2003;22:abstr 1561 (poster presentation).

4 De Gramont A, Banzi M, Navarro M, et al.: Oxaliplatin/5-FU/LV in adjuvant colon cancer: Results of the international randomized mosaic trial. Proc ASCO 2003;22:abstr 1015 (oral presentation).

5 Goldberg RM, Morton RF, Sargent DJ, et al.: N9741: Oxaliplatin (oxal) or CPT-11 + 5-fluorouracil (5-FU)/leucovorin (LV) or oxal + CPT-11 in advanced colorectal cancer (CRC): Initial toxicity and response data from a GI intergroup study. Proc ASCO 2002;21:abstr 511 (oral presentation).
6 Goldberg RM, Morton RF, Sargent DJ, et al.: N9741: Oxaliplatin (oxal) or CPT-11 + 5-fluorouracil (5-FU)/leucovorin (LV) or oxal + CPT-11 in advanced colorectal cancer (CRC): Updated efficacy and quality of life (QOL) data from an intergroup study. Proc ASCO 2003;22:abstr 1009 (oral presentation).

7 Grothey A, Jordan K, Kellner O, et al.: Randomized phase II trial of capecitabine plus irinotecan (CapIri) vs capecitabine plus oxaliplatin (CapOx) as first-line therapy of advanced colorectal cancer. Proc ASCO 2003:22; abstr 1011 (poster presentation).

8 International Multicenter Pooled Analysis of B2 Colon Cancer Trials (IMPACT B2) Investigators; Efficacy of adjuvant fluorouracil and folinic acid in B2 colon cancer. J Clin Oncol 1999;17:1356-1363.

9 Kollmannsberger, C, Rick O, Beyer J, et al.: Activity of oxaliplatin in patients with relapsed or cisplatin-refractory germ cell cancer: A phase II study of the German Testicular Cancer Study Group (GTCSG). Proc ASCO 2001;20:abstr 770 (poster presentation).

10 Louvet C, André T, Lledo G, et al.: Gemcitabine combined with oxaliplatin in advanced pancreatic adenocarcinoma: Final results of a GERCOR multicenter phase II study. J Clin Oncol 2002;20: 1512-1518.
11 Louvet C, Labinaca R, Hammel P, et al.: Gemcitabine versus GEMOX (gemcitabine + oxaliplatin) in non-resectable pancreatic adenocarcinoma: Interim results of the GERCOR/GISCAD Intergroup phase III. Proc ASCO 2003;22:abstr 1004 (oral presentation).

12 Mamounas E, Wienand S, Wolmark N, et al.: Comparative efficacy of adjuvant chemotherapy in patients with Dukes B versus Dukes C colon cancer: results from four national surgical adjuvant breast and bowel project adjuvant studies (C-01, C-02, C03, C-04). J Clin Oncol 1999;17:1349-1355.

13 Rothenberg ML, Oza AM, Burger B, et al. Final results of a phase III trial of 5-FU/leucovorin versus oxaliplatin versus the combination in patients with metastastic colorectal cancer following irinotecan, 5-FU, and leucovorin. Proc ASCO 2003;22:abstr 1011 (oral presentation).

14 Sumpter KA, Harper-Wynne C, Cunningham D, et al.: Randomised, multicentre phase III study comparing capecitabine with fluorouracil and oxaliplatin with cisplatin in patients with advanced oesophagogastro cancer: Confirmation of dose-escalation. Proc ASCO 2003;22:abstr 1031 (poster presentation).

15 Tebutt N, Norman A, Cunningham D, et al.: Randomised, multicentre phase III study comparing capecitabine with fluorouracil and oxaliplatin with cisplatin in patients with advanced oesophago-gastro cancer: Interim analysis. Proc ASCO 2002;21: abstr 523 (oral presentation). 